Département de sciences économiques

Cahier 2004-01

\title{
Deprivation and Social Exclusion
}

BOSSERT, Walter

D'AMBROSIO, Conchita

PERAGINE, Vito 


\section{Département de sciences économiques}

Université de Montréal

Faculté des arts et des sciences

C.P. 6128, succursale Centre-Ville

Montréal (Québec) H3C 3J7

Canada

http://www.sceco.umontreal.ca

SCECO-information@UMontreal.CA

Téléphone : (514) 343-6539

Télécopieur : (514) 343-7221

Ce cahier a également été publié par le Centre interuniversitaire de recherche en économie quantitative (CIREQ) sous le numéro 02-2004.

This working paper was also published by the Center for Interuniversity Research in Quantitative Economics (CIREQ), under number 02-2004.

ISSN 0709-9231 


\title{
Deprivation and Social Exclusion*
}

\author{
WALTER BOSSERT \\ Département de Sciences Economiques and CIREQ, Université de Montréal \\ walter.bossert@umontreal.ca \\ Conchita D'Ambrosio \\ Università di Milano-Bicocca and DIW Berlin \\ conchita.dambrosio@uni-bocconi.it \\ Vito Peragine \\ Università di Bari \\ v.peragine@dse.uniba.it
}

February 2004

* We thank the Social Sciences and Humanities Research Council of Canada, the Fonds pour la Formation de Chercheurs et l'Aide à la Recherche of Québec, the Università Bocconi, ricerca di base "Social Exclusion and Social Distance," the EU TMR network "Livin Tax" (Contract no. ERBFMRXCT980248) and the Ministero dell'Università e della Ricerca Scientifica e Tecnologica, Italy (research project on "The Analysis and Measurement of Freedom") for financial support. The paper was presented at Hitotsubashi University, the Università di Firenze, the Università di Milano, the 2003 Meeting of the Società Italiana degli Economisti in Salerno and the 2003 Meeting of the Società Italiana di Economia Pubblica in Pavia. 


\section{Abstract}

Social exclusion manifests itself in the lack of an individual's access to functionings as compared to other members of society. Thus, the concept is closely related to deprivation. We view deprivation as having two basic determinants: the lack of identification with other members of society and the aggregate alienation experienced by an agent with respect to those with fewer functioning failures. We use an axiomatic approach to characterize classes of deprivation and exclusion measures and apply some of them to EU data for the period from 1994 to 2000. Journal of Economic Literature Classification No.: D63.

Keywords: Social Exclusion, Deprivation, Equity. 


\section{Introduction}

This paper is concerned with the definition and the measurement of social exclusion and its relation with deprivation. When does an individual suffer from deprivation or from social exclusion? What is the level of social exclusion in a given society? Can we say that in the UK there is more social exclusion than in Italy? Qusetions such as these form the main motivation of our study.

In the Treaty of Amsterdam, signed in 1997, the EU included the reduction of social exclusion among its objectives. During the Lisbon European Council of March 2000, the Union formulated the goal "to become the most competitive dynamic knowledge-based economy in the world capable of sustainable economic growth with more and better jobs and greater social cohesion" (European Council, 2000). Hence, social exclusion has gained a primary role in official documents and in the political debate, and it has received a considerable amount of attention among social scientists as well as policy-makers; see, for example, Duffy (1995), Room (1995), Klasen (1998), Rowntree Foundation (1998), Sen (1998), UK House of Commons (1999), Bradshaw, Williams, Levitas, Pantazis, Patsios,

Townsend, Gordon and Middleton (2000), Mejer (2000) and Tsakloglou and Papadopoulos (2001).

Under the heading of social exclusion are included concerns for social phenomena as diverse as poverty, deprivation, low educational attainment, unemployment and other labor market disadvantages, poor housing and lack of access to social and political institutions. Broadly speaking, a person is said to be socially excluded if it is unable to participate in the basic economic and social activities of the society it lives in. Starting from this general idea, a number of different conceptualizations of social exclusion have been proposed in the literature; see, for instance, Atkinson, Cantillon, Marlier and Nolan (2002). The basic elements characterizing this phenomenon include multi-dimensional deprivation, relativity and dynamic considerations. Social exclusion is a multi-dimensional concept that covers economic, social and political aspects: it deals with the failure to attain adequate levels of various functionings (Sen, 1985) that are deemed valuable. Social exclusion is a relative concept in the sense that an individual can be socially excluded only in comparison with other members of a society; hence there is no 'absolute' social exclusion, and an individual can be declared as socially excluded only with respect to the society it is considered to be a member of. An additional relative feature is that it depends on the extent to which an individual is able to associate and identify with others. Social exclusion has important dynamic aspects because it can be viewed as persistent deprivation: an individual can 
become socially excluded if its condition of deprivation is persistent or worsens over time.

On the basis of these properties, we can identify some similarities and differences to the related concepts of inequality and poverty. The multi-dimensionality of social exclusion makes it fundamentally different from income (or consumption) inequality and poverty. In contrast to multi-dimensional inequality, which is a measure of the dispersion in a multi-dimensional distribution of quantities (consumption or functionings) for different individuals (Tsui, 1999), there is an asymmetry in social exclusion: an individual experiences a lack of access to a set of relevant functionings only in relation to those with fewer restrictions and, thus, social exclusion does not necessarily depend on the dispersion in the entire distribution. A measure of multidimensional poverty typically specifies a poverty threshold for each functioning, then looks at the shortfalls of different individuals from the threshold levels of each functioning, and finally aggregates these shortfalls into an overall index of poverty (Bourguignon and Chakravarty, 2003). Thus, both multidimensional poverty and social exclusion deal with functioning failures. However, unlike poverty measurement where a poverty line is used to separate the poor from the non-poor, there is no analogous concept in the measurement of social exclusion due to its relative nature.

Social exclusion is characterized by the persistence of the situation of deprivation over time and, therefore, its measurement requires the inclusion of time as an important variable (see also Atkinson, 1998). In accordance with this view, we define individual exclusion as individual deprivation over time, and social exclusion is obtained as an aggregate of the individual exclusion measures. As is the case for Chakravarty and D'Ambrosio (2003), we employ the axiomatic method. While their approach and ours exhibit some similarities in the way multi-dimensional deprivation is modelled, there are substantial differences in how relative and dynamic aspects are taken into consideration.

We consider the problem of analyzing the foundations of social exclusion measurement an important task because of the public-policy relevance of the issue. Thus, both providing a rigorous conceptual foundation and giving some guidance as to the application of the concepts suggested here are of importance. As a consequence, the purpose of this paper is to combine a sound theoretical investigation with an empirical analysis. This is accomplished by applying some of the measures proposed in the axiomatic part of the paper to recent EU data.

The remainder of the paper is organized as follows. The next section introduces the formal framework for measuring individual deprivation, followed by an extension to aggregate deprivation and individual and aggregate exclusion. In the third section we describe the empirical results for data in EU member states covering the period 1994 to 
2000. Section 4 concludes.

\section{Deprivation and exclusion}

We use $\mathbb{N}$ to denote the set of all positive integers and $\mathbb{R}\left(\mathbb{R}_{++}\right)$denotes the set of all (all positive) real numbers. $\mathbb{Q}_{+}$is the set of all non-negative rational numbers. For a non-empty and finite set $M \subseteq \mathbb{N}$, the set $\mathbb{Q}_{+}^{M}$ is the set of $|M|$-dimensional vectors of non-negative rational numbers whose components are labelled by the elements in $M$. Furthermore, we define $\mathcal{N}=\mathbb{N} \backslash\{1\}$. $\mathcal{P}$ is the set of all finite subsets of $\mathbb{N}$ with at least two elements. For $n \in \mathbb{N}, \mathbf{1}_{n}$ is the vector consisting of $n$ ones. Agents are indexed by positive integers, and $N \in \mathcal{P}$ is the set of individuals in a society.

We assume that, for each individual, there exists a measure of functioning failure which indicates the degree to which functionings that are considered relevant are not available to the agent. The individual functioning failures constitute the primary inputs for our analysis. As a consequence of this modelling chocie, the multidimensionality aspect of social exclusion is not explicitly taken into consideration because we assume that a first aggregation step has already been performed in order to arrive at this single measure of functioning failure. On the other thand, this means that our approach is very flexible because it is compatible with any way in which this aggregation may be performed. A plausible possibility for such a measure is the number of functioning failures, which is the measure used in our empirical application. However, we use a more general approach that assumes the set of possible values of a measure of functioning failure to be the rational numbers. This is plausible because functioning failures could be partial or the measures could incorporate weights that reflect the relative importance of these functioning failures. Our characterization results remain true if all real numbers are allowed as functioningfailure values but, because the irrational reals are not required, we work with the set of rational numbers.

Our axiomatization proceeds in three steps. First, we characterize a class of measures of individual deprivation based on the individual functioning failures. In a second stage, we move from individual deprivation to individual exclusion. An important aspect that distinguishes exclusion from deprivation is the intertemporal aspect of exclusion. Consequently, our notion of social exclusion is obtained as an aggregate of the levels of deprivation experienced by an individual in each of a given number of periods. In a final step, these individual indicators of exclusion are aggregated across individuals to arrive at a class of measures of exclusion for society as a whole. In all cases, we use the 
arithmetic mean (numerous axiomatic justifications of which can be found in the literature; see, for instance, Aczél, 1966, pp. 239-240) as the requisite aggregator function. As a consequence, the most involved part of our axiomatization is the first step - the characterization of measures of individual deprivation. Once this is accomplished, the intertemporal aggregation and the aggregation across individuals is straightforward.

In each period, the individual deprivation measures can be aggregated across individuals to obtain a social measure of deprivation. Contrary to what one might suspect, an intertemporal aggregate of this measure does not lead to the same measure of social exclusion that is obtained if the order of aggregation is reversed. As will become clear once our class of measures has been defined formally, an intuitive reason why this is the case is a lack of separability due to the influence of the set of those with fewer exclusions on the values of the measures.

\subsection{Individual deprivation}

For an individual $i \in \mathbb{N}, q_{i} \in \mathbb{Q}_{+}$is the functioning failure suffered by $i$ in a given period. In this subsection, we consider individual deprivation in a single period only. In order to keep the notation simple, the period under consideration is not identified explicitly. Let $\Omega=\cup_{N \in \mathcal{P}} \mathbb{Q}_{+}^{N}$. A functioning-failure profile is a vector $q \in \Omega$. Let $q, \bar{q} \in \cup_{N \in \mathcal{P}} \mathbb{Q}_{+}^{N}$ and suppose $M \subset N$ is non-empty. The vector $q_{M} \in \mathbb{Q}_{+}^{M}$ is defined by $q_{M}=\left(q_{i}\right)_{i \in M}$ and, analogously, $q_{-M} \in \mathbb{Q}_{+}^{N \backslash M}$ is $q_{-M}=\left(q_{i}\right)_{i \in N \backslash M}$. Finally, $\left(q_{-M}, \bar{q}_{M}\right) \in \mathbb{Q}_{+}^{N}$ is given by $\left(q_{-M}, \bar{q}_{M}\right)_{i}=q_{i}$ if $i \in N \backslash M$ and $\left(q_{-M}, \bar{q}_{M}\right)_{i}=\bar{q}_{i}$ if $i \in M$.

For $i \in \mathbb{N}$, let $\mathcal{P}_{i} \subseteq \mathcal{P}$ be the set of all $N \in \mathcal{P}$ with $i \in N$, and let $\Omega_{i}=\cup_{N \in \mathcal{P}_{i}} \mathbb{Q}_{+}^{N}$. An individual deprivation index for individual $i \in N$ is a function $D_{i}: \Omega_{i} \rightarrow \mathbb{R}_{+}$. For $N \in \mathcal{P}_{i}$ and $q \in \mathbb{Q}_{+}^{N}, D_{i}(q)$ is the degree of deprivation suffered by individual $i$ in profile $q$. The set of individuals whose functioning failure is lower than that of $i$ in $q$ is $\mathcal{B}_{i}(q)=\left\{j \in N \mid q_{j}<q_{i}\right\}$.

We now formulate some desirable properties of $D_{i}$. The first of these is a normalization axiom. We use zero as the minimal value of the deprivation index and, due to the relative nature of the notion of deprivation, we assume that this minimal value of $D_{i}$ is obtained whenever no-one in society has fewer functioning failures than individual $i$. That is, $D_{i}(q)$ is equal to zero whenever the set $\mathcal{B}_{i}(q)$ is empty. Conversely, we require the degree of individual $i$ 's deprivation to be positive whenever there are people who experience fewer functioning failures than $i$. As a result, our normalization axiom requires that the deprivation of individual $i$ is zero if and only if the set of individuals with fewer functioning 
failures is empty - that is, if and only if $i$ 's functioning failures are minimal within the profile under consideration.

Normalization: For all $q \in \Omega_{i}, D_{i}(q)=0$ if and only if $\mathcal{B}_{i}(q)=\emptyset$.

The following axiom illustrates an important difference between the notion of inequality and that of deprivation. In determining the degree of deprivation suffered by an individual $i$, it seems plausible to assume that $i$ 's deprivation depends only on its own functioning failures and on those of the individuals who have fewer functioning failures than $i$, that is, those in $\mathcal{B}_{i}(q)$. The idea that a person's feeling of deprivation in a society arises out of comparing its situation with those who are better off has first been formulated by Runciman (1966) and then used by Sen (1976). Sen argues that individual $i$ 's level of deprivation is an increasing function of the number of people who are better off than $i$. We adapt this general idea to our framework and assume that the extent to which an individual considers itself deprived does not depend on the situation of individuals who have a degree of functioning failure equal to or exceeding that of the individual itself. Thus, unlike in the case of inequality, there is an asymmetry between those who are better off (in terms of functionings) than an individual $i$ and those who are at most as well-off as $i$ itself.

Focus: For all $N \in \mathcal{P}_{i}$ and for all $q, \bar{q} \in \mathbb{Q}_{+}^{N}$, if $\mathcal{B}_{i}(\bar{q})=\mathcal{B}_{i}(q), \bar{q}_{i}=q_{i}$ and $\bar{q}_{j}=q_{j}$ for all $j \in \mathcal{B}_{i}(q)$, then $D_{i}(\bar{q})=D_{i}(q)$.

As usual, anonymity requires that the identities of the individuals are irrelevant in obtaining a social index. For the individual index $D_{i}$, however, it is clear that individual $i$ itself may (and usually will) play a special role. Thus, the anonymity axiom we employ is restricted to the set of individuals other than $i$ and we obtain the following conditional version.

Conditional anonymity: For all $N \in \mathcal{P}_{i}$, for all $q, \bar{q} \in \mathbb{Q}_{+}^{N}$ and for all $j, k \in N \backslash\{i\}$, if $\bar{q}_{j}=q_{k}, \bar{q}_{k}=q_{j}$ and $\bar{q}_{\ell}=q_{\ell}$ for all $\ell \in N \backslash\{j, k\}$, then $D_{i}(\bar{q})=D_{i}(q)$.

The next axiom is a standard condition in much of economic theory. It is (linear) homogeneity, which requires that if a profile is multiplied by a positive number, then the corresponding level of deprivation is multiplied by the same number. This property ensures that a proportional change in the profile of functioning failures leads to an equiproportional change in individual deprivation. 
Homogeneity: For all $q \in \Omega_{i}$ and for all $\lambda \in \mathbb{Q}_{++}, D_{i}(\lambda q)=\lambda D_{i}(q)$.

Translation invariance imposes a restriction on the response of an index to equal absolute changes in a profile. If the same real number is added to each functioning failure, the value of the deprivation index is unchanged. We employ a stronger axiom that applies to additions of different numbers, provided that the set of individuals with fewer functioning failures than $i$ is unchanged and, moreover, the value added to the functioning failures of those that are equally well or worse off than $i$ is the arithmetic mean of the values added to those in $\mathcal{B}_{i}(e)$.

Strong translation invariance: For all $N \in \mathcal{P}_{i}$, for all $q, \bar{q} \in \mathbb{Q}_{+}^{N}$ and for all $\delta \in \mathbb{Q}^{\mathcal{B}_{i}(q)}$, if $\mathcal{B}_{i}(\bar{q})=\mathcal{B}_{i}(q), \bar{q}_{j}=q_{j}+\delta_{j}$ for all $j \in \mathcal{B}_{i}(q)$ and $\bar{q}_{k}=q_{k}+\frac{1}{\left|\mathcal{B}_{i}(q)\right|} \sum_{j \in \mathcal{B}_{i}(q)} \delta_{j}$ for all $k \in N \backslash \mathcal{B}_{i}(q)$, then $D_{i}(\bar{q})=D_{i}(q)$.

The standard translation-invariance axiom is implied by the above condition; it corresponds to the case where the $\delta_{j}$ are equal for all $j \in \mathcal{B}_{i}(q)$.

Finally, we introduce two proportionality properties. The first of these imposes a restriction on the response of $D_{i}$ when the entire population is replicated for some specific functioning-failure profiles, and the second applies to replications of the set of those who are better off than $i$ for a fixed total population.

Population proportionality: For all $m \in \mathcal{N}$, for all $N, M \in \mathcal{P}_{i}$ such that $N \subset M$ and $|M|=m|N|$, for all $q \in \mathbb{Q}_{+}^{N}$, for all $\bar{q} \in \mathbb{Q}_{+}^{M}$ and for all $k \in N \backslash\{i\}$, if $\mathcal{B}_{i}(\bar{q})=\mathcal{B}_{i}(q)=\{k\}$, $\bar{q}_{k}=q_{k}, q_{j}=q_{i}$ for all $j \in N \backslash\{k\}$ and $\bar{q}_{j}=q_{i}$ for all $j \in M \backslash\{k\}$, then $D_{i}(\bar{q})=D_{i}(q) / m^{2}$. Population proportionality applies to specific situations where the population is replicated by a factor $m$ and the number of individuals with fewer restrictions than $i$ is fixed at one. All other individuals have the same degree of functioning failure as $i$. Under these circumstances, the resulting replicated profile leads to a value of individual deprivation given by the value of the original profile divided by $m^{2}$. The reason why deprivation is divided by $m^{2}$ rather than $m$ is that there are two effects of an increased number of individuals. First, the relative importance of the deprivation caused by the single individual who has fewer functioning failures is diminished. Second, the number of individuals with whom $i$ can identify is multiplied.

Deprivation proportionality: For all $m \in \mathcal{N}$, for all $N \in \mathcal{P}_{i}$, for all $q, \bar{q} \in \mathbb{Q}_{+}^{N}$ and for all $k \in N \backslash\{i\}$, if $\mathcal{B}_{i}(q)=\{k\} \subset \mathcal{B}_{i}(\bar{q}),\left|\mathcal{B}_{i}(\bar{q})\right|=m, \bar{q}_{j}=q_{k}$ for all $j \in \mathcal{B}_{i}(\bar{q}), q_{j}=q_{i}$ for all $j \in N \backslash\{k\}$ and $\bar{q}_{j}=q_{j}$ for all $j \in N \backslash \mathcal{B}_{i}(\bar{q})$, then $D_{i}(\bar{q})=m^{2} D_{i}(q)$. 
Deprivation proportionality applies to situations where, for a fixed population, the number of those with fewer restrictions than $i$ is replicated. Again, all other individuals have the same functioning failure as $i$. Because of the two effects of this replication, deprivation is required to be multiplied by $m^{2}$ as a consequence.

The class of deprivation measures characterized by the above axioms has the following structure. The degree of deprivation for a profile $q$ is obtained as the product of two terms with the following interpretation. The first factor is a multiple of the ratio of the number of agents who have fewer functioning failures than $i$ and the population size. As mentioned earlier, this number is an inverse indicator of agent $i$ 's capacity to identify with other members of society. The second factor is the average of the differences between $q_{i}$ and the functioning failures of all agents in $\mathcal{B}_{i}(q)$. This part captures the aggregate alienation experienced by $i$ with respect to those who are better off.

To the best of our knowledge, this index of individual deprivation has not appeared in the literature before. Although our measure of individual deprivation, reinterpreted in terms of income distributions rather than distributions of functioning failures, resembles that suggested by Yitzhaki (1979), there is an important and substantial difference. Yitzhaki defines what we use as the second factor as the individual deprivation index; see Ebert and Moyes (2000) for a characterization. Thus, taking into consideration the lack of identification in addition to aggregate alienation is what distinguishes our approach from earlier contributions.

Theorem $1 D_{i}$ satisfies normalization, focus, conditional anonymity, homogeneity, strong translation invariance, population proportionality and deprivation proportionality if and only if there exists $\alpha_{i} \in \mathbb{R}_{++}$such that, for all $N \in \mathcal{P}_{i}$ and for all $q \in \mathbb{Q}_{+}^{N}$,

$$
D_{i}(q)= \begin{cases}0 & \text { if } \mathcal{B}_{i}(q)=\emptyset \\ \alpha_{i} \frac{\left|\mathcal{B}_{i}(q)\right|}{|N|^{2}} \sum_{j \in \mathcal{B}_{i}(q)}\left(q_{i}-q_{j}\right) & \text { if } \mathcal{B}_{i}(q) \neq \emptyset\end{cases}
$$

Proof. That the indices defined in the theorem statement possess the required properties is straightforward to verify.

Conversely, suppose $D_{i}$ satisfies the axioms of the theorem statement. First, consider a fixed population $N \in \mathcal{P}_{i}$, and let $q \in \mathbb{Q}_{+}^{N}$. If $\mathcal{B}_{i}(q)$ is empty, normalization immediately implies $D_{i}(q)=0$ as desired. Now suppose that $\mathcal{B}_{i}(q) \neq \emptyset$. By definition of this set, this implies that $q_{i}$ is positive.

Now let $q$ be such that all agents in $\mathcal{B}_{i}(q)$ have a functioning failure of zero. By conditional anonymity, the identities of the individuals in $\mathcal{B}_{i}(q)$ are irrelevant and, therefore, 
we can consider the profile $\left(q_{-\mathcal{B}_{i}(q)}, 0 \mathbf{1}_{\left|\mathcal{B}_{i}(q)\right|}\right)$. By the focus axiom,

$$
D_{i}\left(q_{-\mathcal{B}_{i}(q)}, 0 \mathbf{1}_{\left|\mathcal{B}_{i}(q)\right|}\right)=D_{i}\left(q_{i} \mathbf{1}_{\left|N \backslash \mathcal{B}_{i}(q)\right|}, 0 \mathbf{1}_{\left|\mathcal{B}_{i}(q)\right|}\right) .
$$

Using homogeneity with $\lambda=q_{i}$, it follows that

$$
D_{i}\left(q_{i} \mathbf{1}_{\left|N \backslash \mathcal{B}_{i}(q)\right|}, 0 \mathbf{1}_{\left|\mathcal{B}_{i}(q)\right|}\right)=q_{i} D_{i}\left(\mathbf{1}_{\left|N \backslash \mathcal{B}_{i}(q)\right|}, 0 \mathbf{1}_{\mathcal{B}_{i}(q) \mid}\right) .
$$

Now define

$$
a_{i}\left(\left|\mathcal{B}_{i}(q)\right|,|N|\right)=D_{i}\left(\mathbf{1}_{\left|N \backslash \mathcal{B}_{i}(q)\right|}, 0 \mathbf{1}_{\left|\mathcal{B}_{i}(q)\right|}\right) .
$$

Using (1) and substituting into (2), we obtain

$$
D_{i}\left(q_{-\mathcal{B}_{i}(q)}, 0 \mathbf{1}_{\left|\mathcal{B}_{i}(q)\right|}\right)=q_{i} a_{i}\left(\left|\mathcal{B}_{i}(q)\right|,|N|\right)
$$

Because $\mathcal{B}_{i}(q)$ is non-empty, $a_{i}\left(\left|\mathcal{B}_{i}(q)\right|,|N|\right)$ is positive by normalization.

Now consider an arbitrary profile $q \in \mathbb{Q}_{+}^{N}$. By the focus axiom, we can without loss of generality assume that $q_{k}=q_{i}$ for all $k \in N \backslash \mathcal{B}_{i}(q)$. Construct a profile $\bar{q} \in \mathbb{Q}_{+}^{N}$ by letting $\bar{q}_{j}=q_{j}-q_{j}=0$ for all $j \in \mathcal{B}_{i}(q)$ and $\bar{q}_{k}=q_{i}-\frac{1}{\left|\mathcal{B}_{i}(q)\right|} \sum_{j \in \mathcal{B}_{i}(q)} q_{j}$ for all $k \in N \backslash \mathcal{B}_{i}(q)$. Using strong translation invariance with $\delta_{j}=-q_{j}$ for all $j \in \mathcal{B}_{i}(q)=\mathcal{B}_{i}(\bar{q})$, it follows that $D_{i}(\bar{q})=D_{i}(q)$. Because all agents in $\mathcal{B}_{i}(\bar{q})$ have a functioning failure of zero in $\bar{q},(3)$ implies

$$
\begin{aligned}
D_{i}(q)=D_{i}(\bar{q}) & =\bar{q}_{i} a_{i}\left(\left|\mathcal{B}_{i}(\bar{q})\right|,|N|\right) \\
& =\left(q_{i}-\frac{1}{\left|\mathcal{B}_{i}(q)\right|} \sum_{j \in \mathcal{B}_{i}(q)} q_{j}\right) a_{i}\left(\left|\mathcal{B}_{i}(q)\right|,|N|\right) \\
& =\left(\left|\mathcal{B}_{i}(q)\right| q_{i}-\sum_{j \in \mathcal{B}_{i}(q)} q_{j}\right) \frac{a_{i}\left(\left|\mathcal{B}_{i}(q)\right|,|N|\right)}{\left|\mathcal{B}_{i}(q)\right|} \\
& =\frac{a_{i}\left(\left|\mathcal{B}_{i}(q)\right|,|N|\right)}{\left|\mathcal{B}_{i}(q)\right|} \sum_{j \in \mathcal{B}_{i}(q)}\left(q_{i}-q_{j}\right) \\
& =F_{i}\left(\left|\mathcal{B}_{i}(q)\right|,|N|\right) \sum_{j \in \mathcal{B}_{i}(q)}\left(q_{i}-q_{j}\right),
\end{aligned}
$$

where $F_{i}\left(\left|\mathcal{B}_{i}(q)\right|,|N|\right)=\frac{a_{i}\left(\left|\mathcal{B}_{i}(q)\right|,|N|\right)}{\left|\mathcal{B}_{i}(q)\right|} . F_{i}$ is positive-valued because $a_{i}$ is.

Let $N \in \mathcal{P}_{i}$ be such that $|N|=2$, let $m \in \mathcal{N}$ and let $q, \bar{q}$ be as in the definition of population proportionality. By (4), we have

$$
D_{i}(q)=F_{i}(1,2)\left(q_{i}-q_{k}\right)
$$


and

$$
D_{i}(\bar{q})=F_{i}(1,2 m)\left(q_{i}-q_{k}\right) .
$$

By population proportionality,

$$
F_{i}(1,2 m)\left(q_{i}-q_{k}\right)=\frac{1}{m^{2}} F_{i}(1,2)\left(q_{i}-q_{k}\right)
$$

and, because $q_{k}<q_{i}$, it follows that

$$
F_{i}(1,2 m)=\frac{1}{m^{2}} F_{i}(1,2)
$$

for all $m \in \mathcal{N}$. Thus,

$$
F_{i}(1, n)=\frac{4}{n^{2}} F_{i}(1,2)
$$

for all even $n \in \mathcal{N}$. Now suppose $n \in \mathcal{N}$ is odd. Let $m=2$ and apply population proportionality again to obtain

$$
F_{i}(1,2 n)=\frac{1}{4} F_{i}(1, n) .
$$

Thus,

$$
F_{i}(1, n)=4 F_{i}(1,2 n) .
$$

Because $2 n$ is even, (5) implies

$$
F_{i}(1,2 n)=\frac{1}{n^{2}} F_{i}(1,2)
$$

and, therefore,

$$
F_{i}(1, n)=4 F_{i}(1,2 n)=\frac{4}{n^{2}} F_{i}(1,2)
$$

for all odd $n \in \mathcal{N}$. Thus, letting $\alpha_{i}=4 F_{i}(1,2) \in \mathbb{R}_{++}$, we obtain

$$
F_{i}(1, n)=\alpha_{i} \frac{1}{n^{2}}
$$

for all $n \in \mathcal{N}$.

Let $N \in \mathcal{P}_{i}$ and $m \in \mathcal{N}$ be such that $m \leq n=|N|$ and consider $q, \bar{q}$ as in the definition of deprivation proportionality. Using (4), it follows that

$$
D_{i}(q)=F_{i}(1, n)\left(q_{i}-q_{k}\right)
$$

and

$$
D_{i}(\bar{q})=F_{i}(m, n) m\left(q_{i}-q_{k}\right) .
$$


By deprivation proportionality,

$$
F_{i}(m, n) m\left(q_{i}-q_{k}\right)=m^{2} F_{i}(1, n)\left(q_{i}-q_{k}\right)
$$

and, therefore,

$$
F_{i}(m, n)=m F_{i}(1, n)
$$

Using (6), we obtain

$$
F_{i}(m, n)=\alpha_{i} \frac{m}{n^{2}} .
$$

Substituting into (4) completes the proof.

\subsection{Aggregate deprivation}

An aggregate deprivation measure is a function $\mathbf{D}: \Omega \rightarrow \mathbb{R}_{+}$. We define aggregate deprivation as the arithmetic mean of the individual deprivation levels. This aggregator can be justified by standard sets of axioms characterizing the arithmetic mean; see, for example, Aczél (1966, pp. 239-240). Thus, our aggregate deprivation measure is given by

$$
\mathbf{D}(q)=\frac{1}{|N|} \sum_{i \in N} D_{i}(q)
$$

for all $N \in \mathcal{P}$ and for all $q \in \mathbb{Q}_{+}^{N}$, where $D_{i}$ is an individual deprivation measure as characterized in Theorem 1.

\subsection{Individual exclusion measures}

To incorporate the dynamic aspect of exclusion, we now consider an intertemporal extension of the individual deprivation measures characterized in Theorem 1. Suppose the set of time periods is $T=\{1, \ldots,|T|\}$ with $|T| \in \mathcal{N}$. Let $\Gamma=\cup_{N \in \mathcal{P}}\left(\mathbb{Q}_{+}^{N}\right)^{|T|}$. An intertemporal functioning-failure profile is a vector $\mathbf{q}=\left(q^{1}, \ldots, q^{|T|}\right) \in \Gamma$. Note that, in a given profile, the population is the same in each time period. An exclusion measure for individual $i$ is a mapping $E_{i}: \Gamma \rightarrow \mathbb{R}_{+}$that assigns $i$ 's level of exclusion to each profile of intertemporal functioning failures.

We employ the average deprivation over the periods under consideration as our measure of individual exclusion. That is, we define

$$
E_{i}(\mathbf{q})=\frac{1}{|T|} \sum_{t \in T} D_{i}\left(q^{t}\right)
$$

for all $N \in \mathcal{P}_{i}$ and for all $\mathbf{q} \in\left(\mathbb{Q}_{+}^{N}\right)^{|T|}$, where $D_{i}$ is an individual deprivation measure as characterized in Theorem 1. 


\subsection{Social exclusion measures}

The final step in our derivation of a class of social-exclusion measures consists of aggregating the individual measures defined in the previous subsection across individuals. For that purpose, we define an aggregate exclusion measure as a function $\mathbf{E}: \Gamma \rightarrow \mathbb{R}_{+}$that assigns an aggregate level of exclusion to each profile of intertemporal functioning failures. Again using the arithmetic mean as our aggregation procedure, we can define our measure as

$$
\mathbf{E}(\mathbf{q})=\frac{1}{|N|} \sum_{i \in N} E_{i}(\mathbf{q})=\frac{1}{|N|} \sum_{i \in N} \frac{1}{|T|} \sum_{t \in T} D_{i}\left(q^{t}\right)
$$

for all $N \in \mathcal{P}$ and for all $\mathbf{q} \in\left(\mathbb{Q}_{+}^{N}\right)^{|T|}$, where $D_{i}$ is an individual deprivation measure as characterized in Theorem 1.

Clearly, the minimal level of social exclusion is equal to zero and attained in the case where everyone has the same index of functioning failure in all periods, that is, in the case of complete equality. This is true for measures of inequality and for those of polarization as well if incomes are reinterpreted as indices of functionings. In contrast, the maximal level of inequality is attained for a distribution where one individual has access to all functionings and everyone else has the maximal possible functioning failure. Furthermore, Esteban and Ray's (1994) measure of polarization is maximal for a distribution where half of the population have full functioning failure whereas the other half have no functioning failures. Interestingly, our measures of social deprivation and social exclusion are not maximal for either of those distributions. In fact, it need not even be the case that maximal functioning failure and zero functioning failure are the only values attained in a distribution in order for this distribution to have maximal social deprivation (or exclusion).

To illustrate, we provide an example. For simplicity, we consider social deprivation only; the example is easily extended to measures of social exclusion.

Suppose that $N=\{1, \ldots, 13\}, \alpha_{i}=1$ for all $i \in N$ and the maximal possible functioning failure is equal to one. Consider a distribution $q \in \mathbb{Q}_{+}^{N}$ such that $q_{1}=\ldots=q_{4}=1$, $q_{5}=q_{6}=1 / 2, q_{7}=1 / 4$ and $q_{8}=\ldots=q_{13}=0$. It is straightforward to verify that $\mathbf{D}(q)=333.5 / 13^{3}$ and that this exceeds the value of $\mathbf{D}$ for any 13-dimensional distribution whose components assume the values zero and one only.

\section{An application to EU countries}

Social exclusion has recently become one of the main concepts in social-policy debates in EU countries. With the Treaty of Amsterdam, which came into force in 1999, the EU 
has, indeed, enlarged its objectives to include the combating of social exclusion among its members. The purpose of this section is to illustrate the social exclusion measure, $\mathbf{E}$ in (8), and the deprivation measure, $\mathbf{D}$ in (7), proposed in the paper using the European Community Household Panel (ECHP). In the application, we let the constant $\alpha_{i}$ be equal to one for all $i$. We base our analysis on all the waves that are currently available of ECHP, which cover the period from 1994 to 2000. The surveys are conducted at a European national level. The ECHP is an ambitious effort at collecting information on the living standards of the households of the EU member states using common definitions, information collection methods and editing procedures. It contains detailed information on incomes, socio-economic characteristics, housing amenities, consumer durables, social relations, employment conditions, health status, subjective evaluation of well-being, etc. Of the 15 EU member states, we could not consider Austria, Finland, Luxembourg and Sweden since the data for these countries were not available for all the waves. For similar reasons we had to exclude Germany and the UK. In particular, the ECHP surveys of these countries were substituted by national surveys, SOEP and BHPS respectively, that did not collect information on all the variables considered in our application. Information has been collected at the individual or the household level depending on the variable, but the unit of our analysis is the individual. The calculation uses required sample weights and, since we are interested in analyzing the persistence of deprivation, we considered only individuals that were interviewed in all the seven waves. In ECHP a person's life has been measured along the following domains: financial difficulties, basic needs and consumption, housing conditions, durables, health, social contacts and participation, and life satisfaction.

For the choice of the non-monetary indicators to be considered for measuring social exclusion and deprivation with the ECHP, we follow the suggestions of Eurostat (2000) and analyze the well-being of EU societies focusing on the 14 non-monetary variables proposed there. These are the following:

- Financial difficulties: 1. Proportion of persons living in households that have great difficulties in making ends meet; 2. Proportion of persons living in households that are in arrears with (re)payment of housing and/or utility bills;

- Basic necessities: 3. Proportion of persons living in households which cannot afford meat, fish or chicken every second day; 4. Proportion of persons living in households which cannot afford to buy new clothes; 5 . Proportion of persons living in households which cannot afford a week's holiday away from home; 
- Housing conditions: 6. Proportion of persons living in the accommodation without a bath or shower; 7. Proportion of persons living in the dwelling with damp walls, floors, foundations, etc.; 8. Proportion of persons living in households which have a shortage of space;

- Durables: 9. Proportion of persons not having access to a car due to a lack of financial resources in the household; 10. Proportion of persons not having access to a telephone due to a lack of financial resources in the household; 11. Proportion of persons not having access to a color TV due to a lack of financial resources in the household;

- Health: 12. Proportion of persons (over 16) reporting bad or very bad health;

- Social contact: 13. Proportion of persons (over 16) who meet their friends or relatives less often than once a month (or never);

- Dissatisfaction: 14. Proportion of persons (over 16) being dissatisfied with their work or main activity.

Note that there are 15 non-monetary indicators recommended in Eurostat (2000). We decided to drop the one belonging to the health domain, namely the proportion of people that were severely hampered in their daily activities by long-lasting health problems, since there was a considerable discrepancy across the ECHP waves for this indicator.

We calculate $\mathbf{E}$ and $\mathbf{D}$ separately for two sets of indicators $V_{1}$ and $V_{2}$, where $V_{1}$ includes the indicators in the domains of financial difficulties, basic necessities, housing conditions and durables, and $V_{2}$ includes the remaining indicators. The reason for separate calculations is that for indicators covered under $V_{1}$ we have household level information, whereas for the indicators in $V_{2}$ the available information is at the individual level, with the additional constraint that the minimum age of the reportee is 16 .

Numerical estimates of social exclusion as measured by $\mathbf{E}$ for the EU member states are reported in Table 1, the values being plotted in Figures 1 and 2. The first column of the table gives the names of the countries for whom the required information was available. In column 2 we present, for each country, the estimates for $V_{1}$ (values plotted in Figure 1 ) while column 3 gives the analogous values for $V_{2}$ (values plotted in Figure 2). Several interesting features emerge from Table 1. Portugal is the most excluding country followed by Greece. At a distance we observe the other two Southern European countries, namely Spain and Italy. The value of $\mathbf{E}$ for Ireland is slightly higher than the one for Italy. If 
we consider the ranking of countries from high to low exclusion, then an unambiguous sequence is Portugal, Greece, Spain, Ireland, Italy, France, Belgium, the Netherlands and Denmark. In $V_{2}$ as well, Portugal is the member state with maximum exclusion, Italy has the second worst off position and Ireland performs the best by showing the lowest values. Denmark and the Netherlands show values higher than Ireland but lower than all other member states. The other countries, namely Greece, Spain, France and Belgium are divided into two groups with Belgium belonging to one separate group with relatively lower values of exclusion. Finally, except for Portugal, the ranking of countries by any measure in $V_{2}$ is different from that in $V_{1}$.

Estimates of $\mathbf{D}$ are reported in Tables 2 (variables included in $V_{1}$ ) and 3 (variables included in $V_{2}$ ), and plotted in Figures 3 and 4 respectively. In the first column of the tables the names of the countries are indicated, while in all the following columns the values that the deprivation index assumes over the years are reported. In the deprivation measure, as opposed to social exclusion, we do not consider persistence in the deprivation state. In other words, the deprivation index is the same as the social exclusion one, the only difference being that the individual deprivation variable in the social exclusion measure is the sum (in this application, without discounting) of the individual deprivation variables of the seven waves. Persistence in the deprivation state is a key variable in understanding the different performance of EU member states in the two measures suggested in this paper. We focus first on the estimates of deprivation for $V_{1}$ (Figure 3). The countries appear to be grouped into three classes according to the level of deprivation reached: Portugal and Greece into the first; Ireland, Spain and Italy into the second; all the remaining countries into the remaining class. In all the years we observe a descending trend and convergence over time in particular of the second and third group that present values of deprivation much more similar in the last wave than they were in the first. Based on these observations on deprivation, we can re-read the values of the measures of social exclusion. We notice the three classes, definitely Portugal and Greece, perform very differently than all other countries, but the position of France is now more ambiguous being in between the second and third class. Portugal and Greece present a greater dissimilarity in social exclusion than in deprivation in all the years considered. This fact is caused by the higher persistence in the deprivation state that individuals face in Portugal than in Greece. In other words, in each period the percentage of the population that is deprived is slightly higher in Portugal than in Greece, but in the latter it is easier for individuals to escape from the deprivation state than it is in the former. Hence, the individuals deprived that we observe in each period vary more over time in Greece than in Portugal. 
In deprivation for $V_{2}$ (Figure 4), we do not observe nor convergence over time, neither a common descending trend. In the first two waves Italy was the most deprived country, but a drop in the value observed starting from the third wave associated with lower persistence led Portugal to be more socially excluded than Italy.

\section{Conclusion}

This paper investigates deprivation and social exclusion both from a theoretical perspective and from an empirical viewpoint. We present an axiomatic aproach that identifies attractive measures of social exclusion and clarifies their possible relationship to the measurement of deprivation. In addition, we apply our measures to recent EU data. We conclude the paper with a few suggestions for future work.

On the theoretical side, it would be interesting to investigate the interplay between measures of social exclusion and other social indicators such as mobility and polarization; see, for instance, Wolfson (1994), Esteban and Ray (1994, 1999), Akerlof (1997), Fields and Ok (1999), Wang and Tsui (2000) and Duclos, Esteban and Ray (2002). Furthermore, systematic approaches to ethically significant exclusion measurement analogous to those employed in the measurement of inequality (see, for example, Kolm, 1969, Atkinson, 1970, Sen, 1973, and Ebert, 1987) could be developed.

From an empirical perspective, the analysis carried out with our data could be complement by studies employing observations from other countries. Moreover, a comparative analysis of different measures would be an interesting task.

\section{References}

Aczél, J. (1966): Lectures on Functional Equations and Their Applications, Academic Press, New York.

Akerlof, G.A. (1997): "Social Distance and Social Decision," Econometrica, 65, 10051027.

Atkinson, A.B. (1970): "On the Measurement of Inequality," Journal of Economic Theory, $2,244-263$.

Atkinson, A.B. (1998): "Social Exclusion, Poverty and Unemployment," in: A.B. Atkinson and J. Hills (eds.), Exclusion, Employment and Opportunity, CASE/4, Centre for Analysis of Social Exclusion, London School of Economics, 1-20. 
Atkinson, A.B., B. Cantillon, E. Marlier and B. Nolan (2002): Social Indicators: The EU and Social Inclusion, Oxford University Press, Oxford.

Bourguignon, F. and S.R. Chakravarty (2003): "The Measurement of Multidimensional Poverty," Journal of Economic Inequality, 1, 25-49.

Bradshaw, J., Williams, J., Levitas, R., Pantazis, C., Patsios, D., Townsend, P., Gordon, D. and S. Middleton (2000): "The Relationship Between Poverty and Social Exclusion in Britain," Paper presented at the 26th General Conference of the International Association for Research in Income and Wealth, Cracow, Poland.

Chakravarty, S.R. and C. D'Ambrosio (2003): "The Measurement of Social Exclusion," DIW Berlin, Discussion Paper.

Duclos, J.-Y., J.-M. Esteban and D. Ray (2002): "Polarization: Concepts, Measurement, Estimation," mimeo.

Duffy, K. (1995): "Social Exclusion and Human Dignity in Europe," Council of Europe, Strasbourg.

Ebert, U. (1987): "Size and Distribution of Incomes as Determinants of Social Welfare," Journal of Economic Theory, 41, 23-33.

Ebert, U. and P. Moyes (2000), "An Axiomatic Characterization of Yitzhaki's Index of Individual Deprivation," Economics Letters, 68, 263-270.

Esteban, J.-M. and D. Ray (1994): "On the Measurement of Polarization," Econometrica, $62,819-851$.

Esteban, J.-M. and D. Ray (1999): "Conflict and Distribution," Journal of Economic Theory, 87, 379-415.

European Council (2000): "Presidency Conclusions," Lisbon European Council of 23 and 24 March 2000.

Eurostat (2000): "European Social Statistics. Income, Poverty and Social Exclusion," THEME 3, Population and Social Conditions, Luxembourg.

Fields, G.S. and E.A. Ok (1999): "The Measurement of Income Mobility: An Introduction to the Literature," in: J. Silber (ed.), Handbook of Income Inequality Measurement, Kluwer, Dordrecht, 557-596.

Klasen, S. (1998): "Social Exclusion and Children in OECD Countries: Some Conceptual Issues," Centre for Educational Research and Innovation, OECD. 
Kolm, S.-C. (1969): "The Optimal Production of Social Justice," in: J. Margolis and S. Guitton (eds.), Public Economics, Macmillan, London, 45-200.

Mejer, L. (2000): "Statistics on Social Exclusion: The EU Methodological Approach," Eurostat, Unit E2 Living Conditions.

Room, G. (1995): Beyond the Threshold: The Measurement and Analysis of Social Exclusion, Policy Press, Bristol.

Rowntree Foundation (1998): "The Report of Key Indicators of Poverty and Social Exclusion," www.parliament.the-stationery-office.co.uk/pa/cm199798/cmselect/777/8060303.htm. Runciman, W.G. (1966): Relative Deprivation and Social Justice, Routledge, London. Sen, A.K. (1973): On Economic Inequality, Oxford University Press, Oxford.

Sen, A.K. (1976): "Poverty: An Ordinal Approach to Measurement," Econometrica, 44, 219-231.

Sen, A.K. (1985): Commodities and Capabilities, North Holland, Amsterdam.

Sen, A.K. (1998): "Social Exclusion and Economic Measurement," paper presented at the 25th General Conference of the International Association for Research in Income and Wealth, Cambridge, UK.

Tsakloglou, P. and F. Papadopoulos (2001): "Identifying Population Groups at High Risk of Social Exclusion," forthcoming in: R. Muffels and P. Tsakloglou (eds.), Social Exclusion in European Welfare States, Edward Elgar, Cheltenham.

Tsui, K.-Y. (1999): "Multidimensional Inequality and Multidimensional Generalized Entropy Measures: An Axiomatic Derivation," Social Choice and Welfare, 16, 145-157.

UK House of Commons (1999): "Poverty and Social Exclusion (National Strategy) Bill," www.parliament.the-stationery-office.co.uk/pa/cm199899/cmbills/045/1999045.htm.

Wang, Y.-Q. and K.-Y. Tsui (2000): "Polarization Orderings and New Classes of Polarization Indices," Journal of Public Economic Theory, 2, 349-363.

Wolfson, M.C. (1994): "When Inequalities Diverge," American Economic Review, 84, $353-358$.

Yitzhaki, S. (1979): "Relative Deprivation and the Gini Coefficient," Quarterly Journal of Economics, 93, 321-324. 


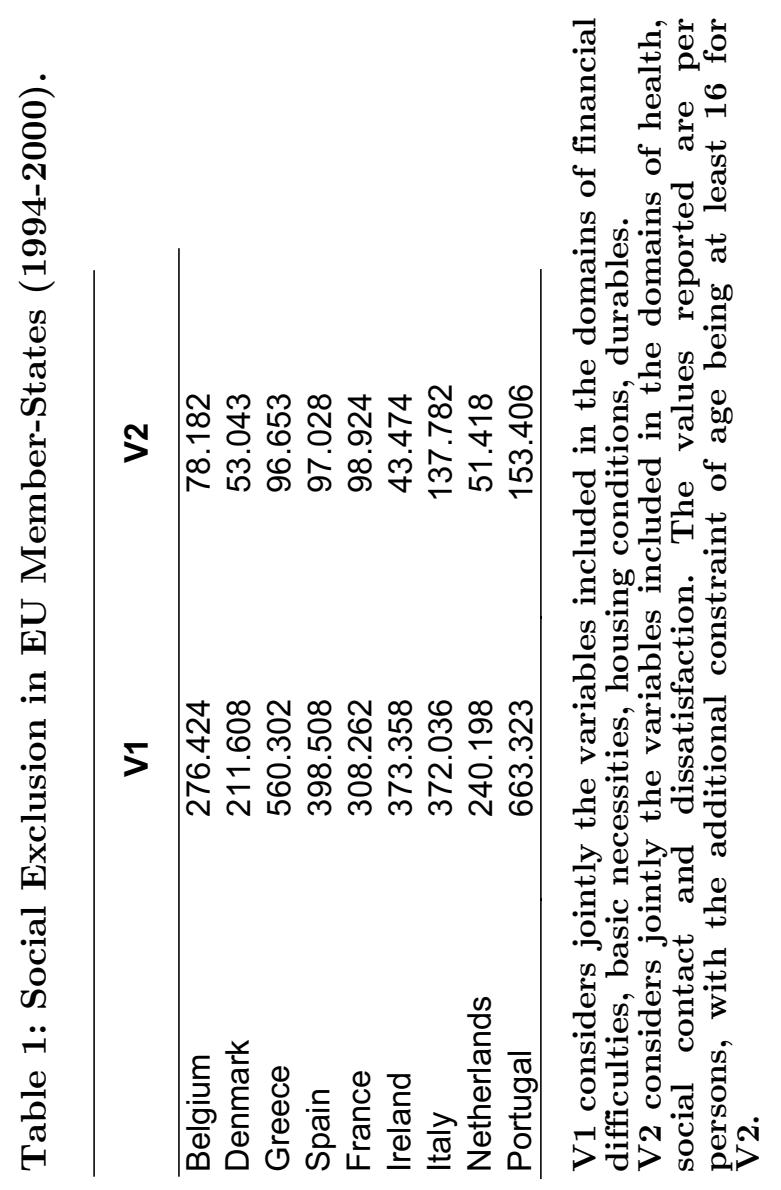



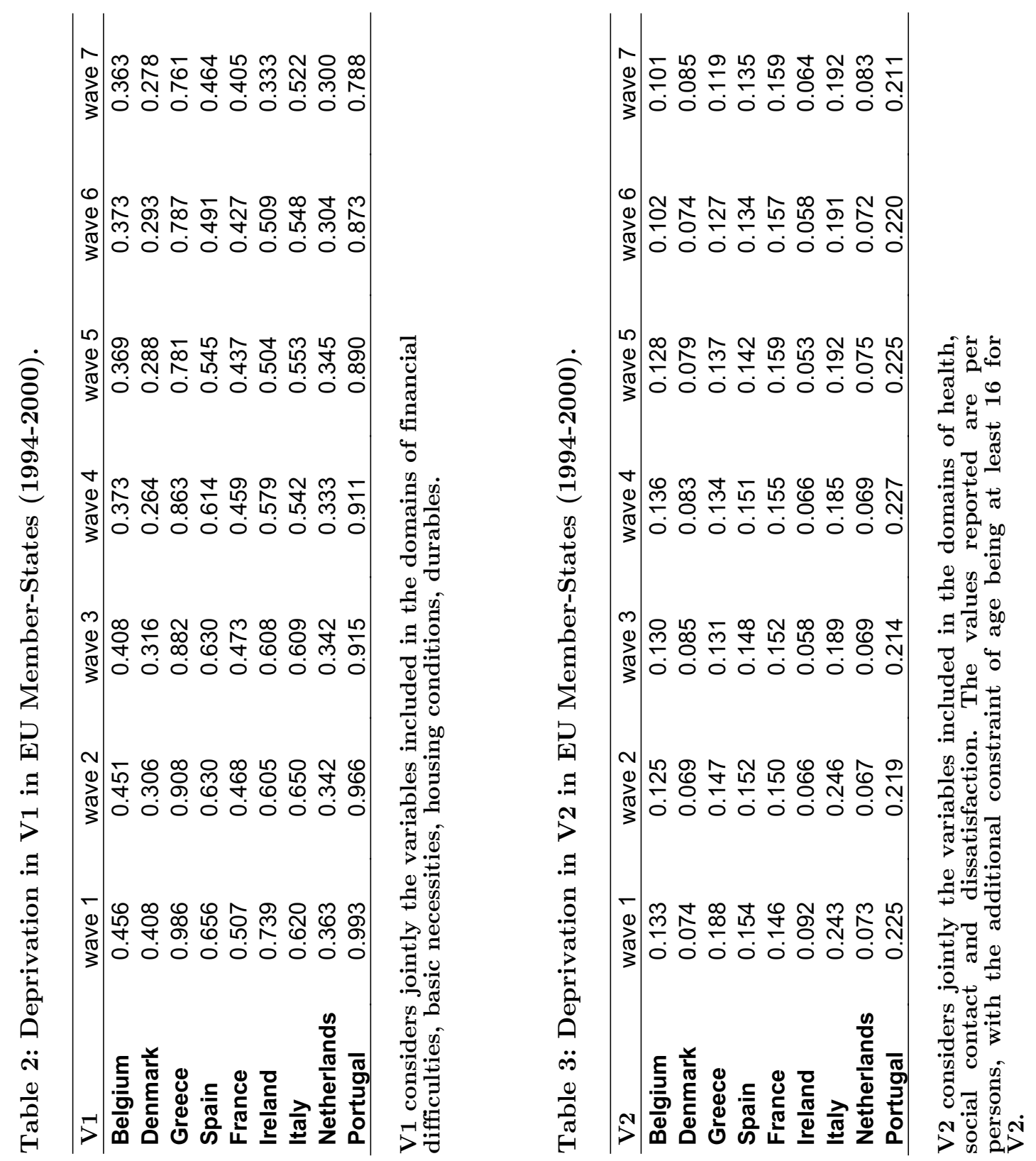
Fure 1: Social Exclusion in EU Member-States (1994-2000), V ${ }_{1}$.

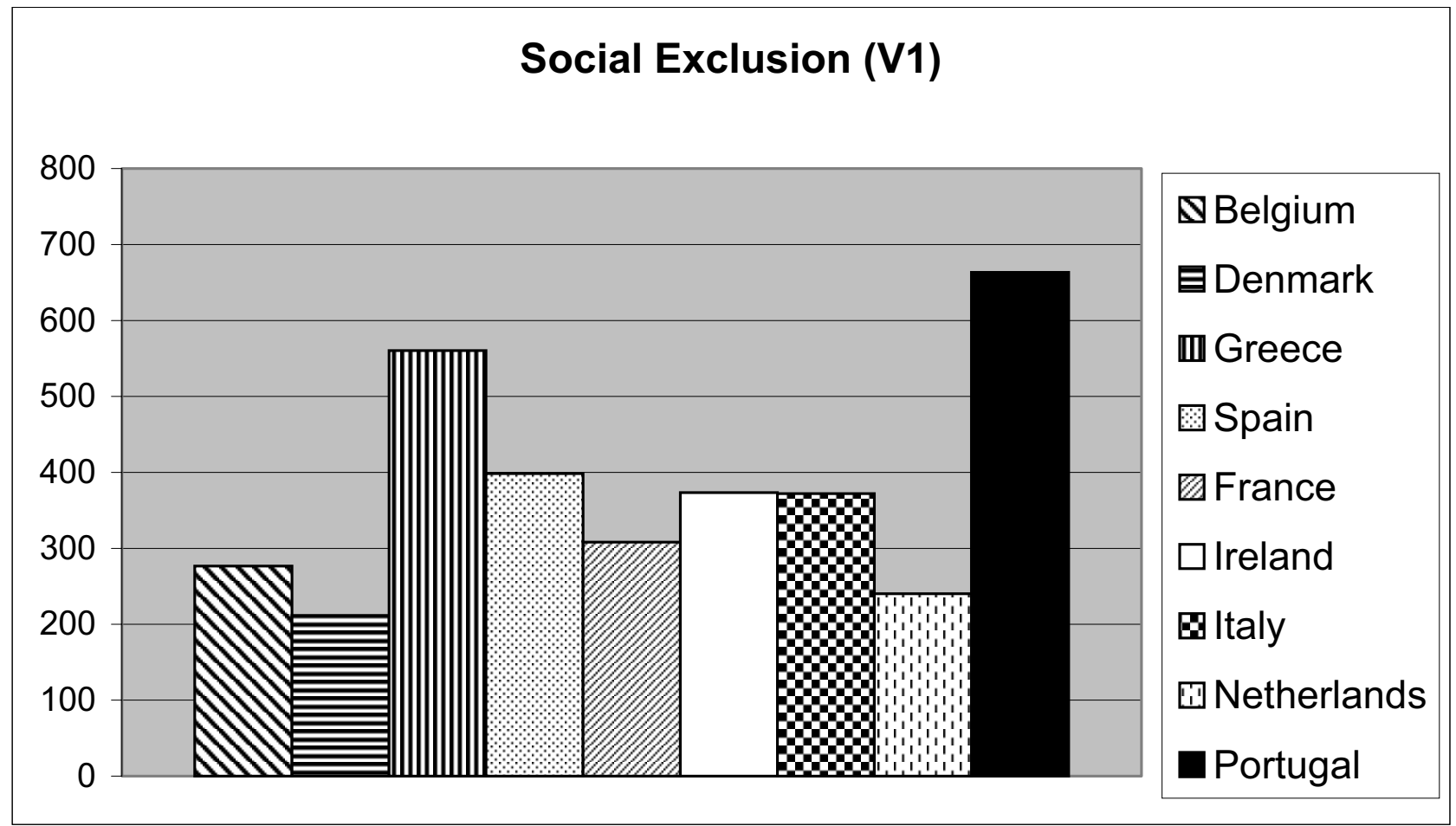

Fure 2: Social Exclusion in EU Member-States (1994-2000), $\mathrm{V}_{2}$.

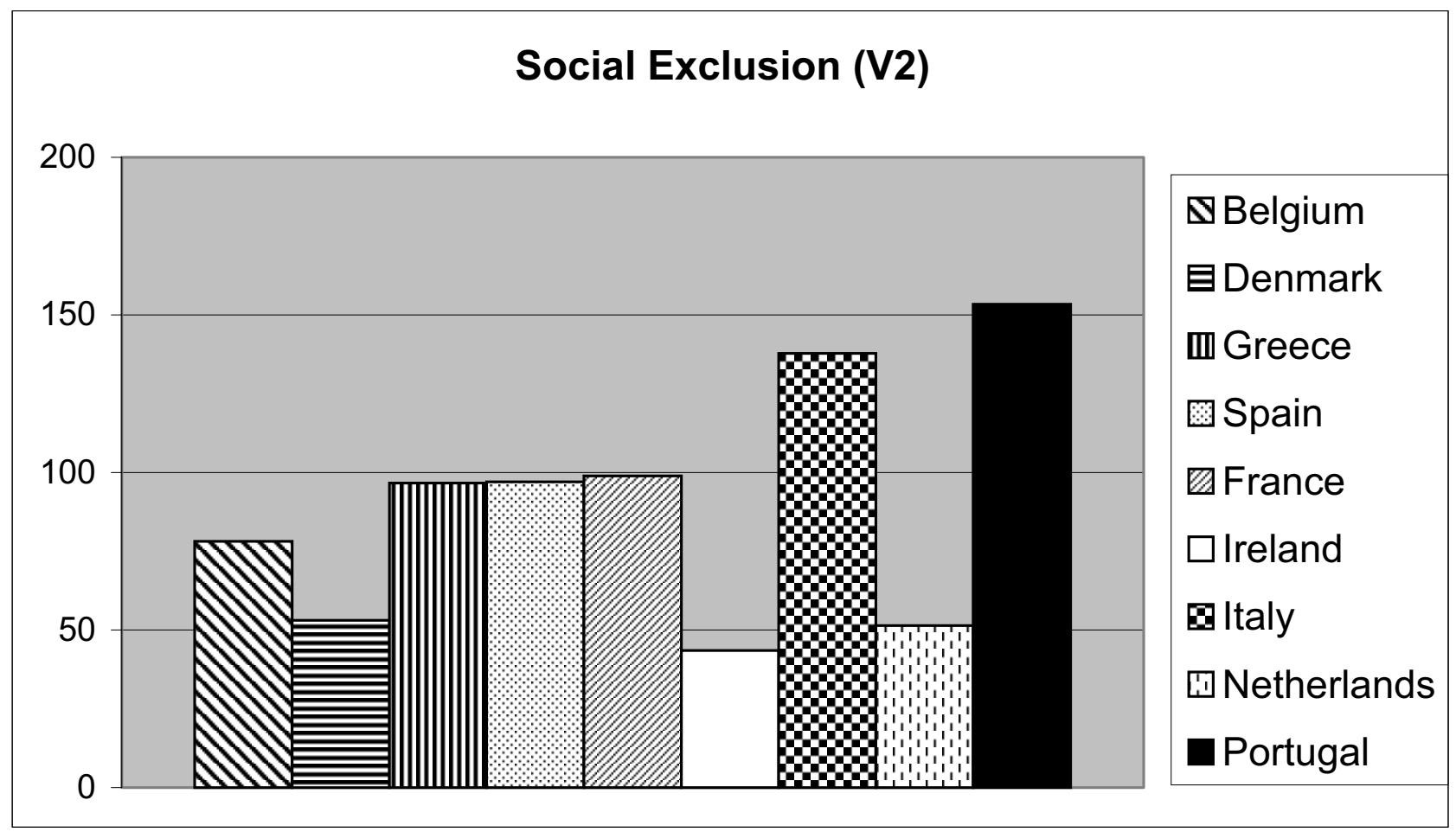


Foure 3: Deprivation in EU Member States (1994-2000), V ${ }_{1}$.

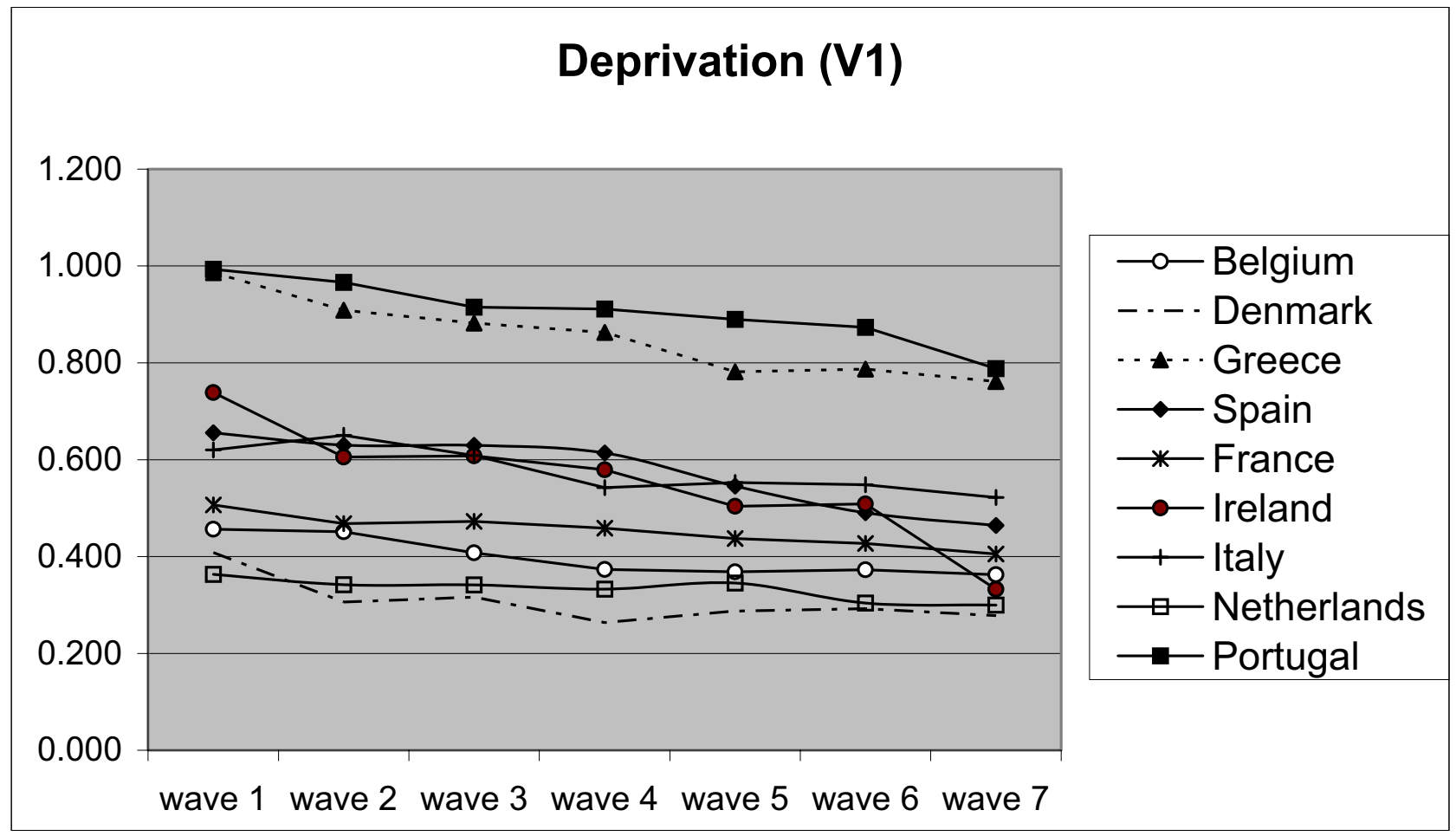

Fure 4: Deprivation in EU Member States (1994-2000), $\mathrm{V}_{2}$.

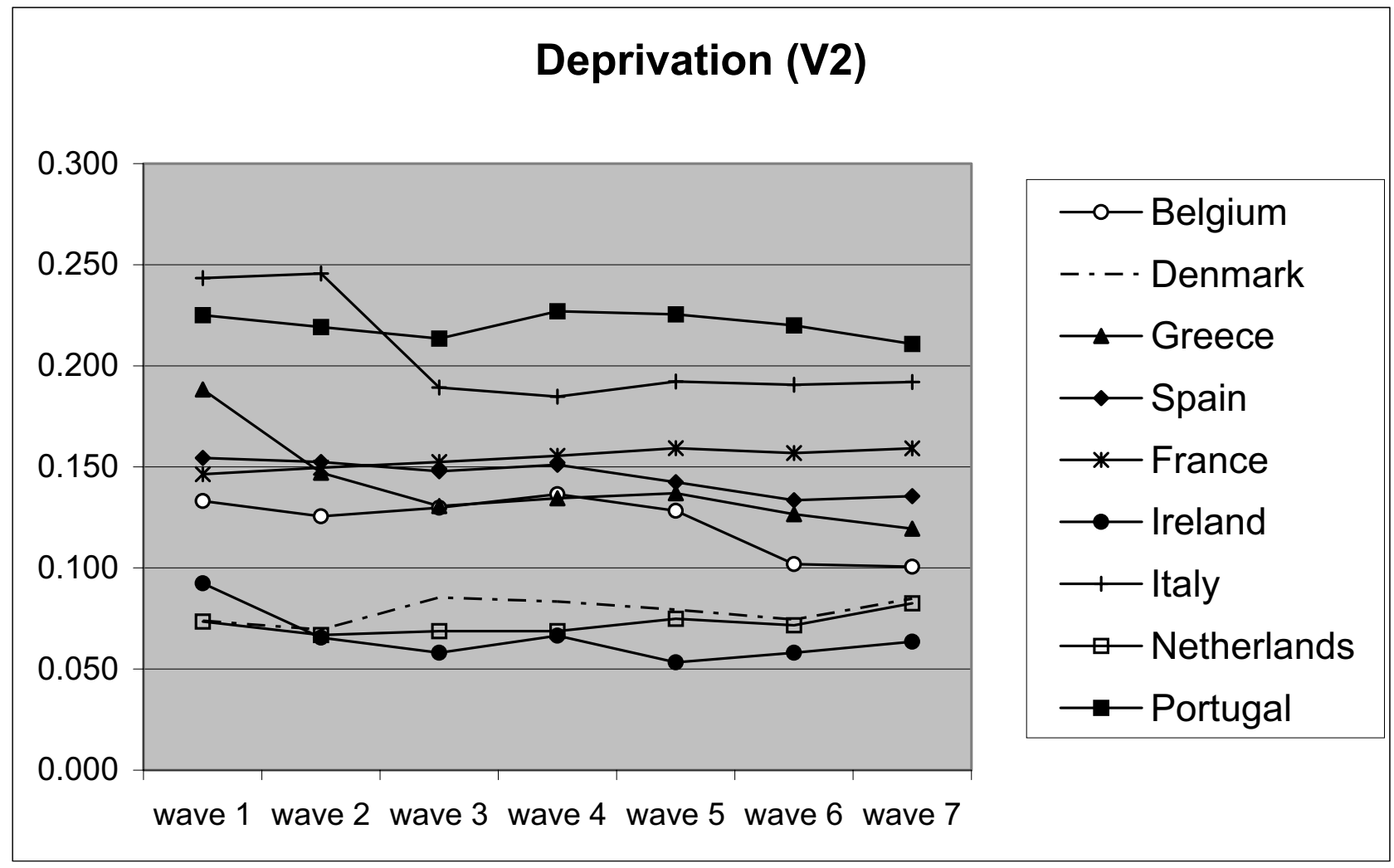

